\title{
DOES PORCINE OOCYTES MATURATION IN VITRO IS REGULATED BY GENES INVOLVED IN TRANSFORMING GROWTH FACTOR BETA RECEPTOR SIGNALING PATHWAY?
}

\author{
Joanna BUDNA ${ }^{1}$, Piotr CELICHOWSKI ${ }^{1}$, Paresto KARIMI ${ }^{2}$, Wiesława KRANC ${ }^{2}$, \\ Artur BRYJA², Sylwia CIESIÓŁKA ${ }^{1}$, Marta RYBSKA ${ }^{3}$, Sylwia BORYS ${ }^{2}$, \\ Michal JESETA ${ }^{4}$, Dorota BUKOWSKA ${ }^{3}$, Paweł ANTOSIK ${ }^{3}$, \\ Klaus P. BRÜSSOW ${ }^{2}$, Małgorzata BRUSKA ${ }^{2}$, Michał NOWICKI ${ }^{1}$, \\ Maciej ZABEL ${ }^{1,5}$, Bartosz KEMPISTY ${ }^{1,2}$ \\ ${ }^{1}$ Department of Histology and Embryology, Poznan University of Medical \\ Sciences, Poland \\ ${ }^{2}$ Department of Anatomy, Poznan University of Medical Sciences, Poland \\ ${ }^{3}$ Institute of Veterinary Sciences, Poznan University of Life Sciences, Poland \\ ${ }^{4}$ Department of Obstetrics and Gynecology, University Hospital and Masaryk \\ University, Brno, Czech Republic \\ ${ }^{6}$ Department of Histology and Embryology, Wroclaw Medical University, Poland
}

DOI: $10.1515 / \mathrm{acb}-2017-0001$

Summary: The oocyte growth and development in follicular environment are substantially accompanied by surrounding somatic cumulus (CCs) and granulosa cells (GCs). During these processes, the mammalian gametes reach full maturational stage and may be further successfully fertilized by single spermatozoon. These unique mechanisms are regulated by expression of clusters of genes and their biochemical signaling pathways.

In this article we described differential expression pattern of transforming growth factor beta (TGFB) gene superfamily in porcine oocytes before and after in vitro maturation (IVM).

We performed Affymetrix ${ }^{\circledR}$ microarray assays to investigate the TGFB-related genes expression profile in porcine immature oocytes and gametes cultured for $44 \mathrm{~h}$ in vitro.

In results we found 419 different genes, 379 genes with lower expression, and 40 genes characterized by increased RNA profile. Moreover, significant up-regulation of 6 genes belonging to TGFB signaling pathway such as: TGFBR3, SMAD4, FOS, KLF10, ID1, MAP3K1 in immature porcine oocytes (before IVM), was also observed.

It may be suggested that genes involved in TGFB-related signaling pathway are substantially regulated before IVM. Furthermore, these genes may play a significant role during early stages of 
nuclear and/or cytoplasmic porcine oocytes maturation. The investigated transcripts may be also recommended as the markers of oocytes maturational capability in pigs.

Keywords: pig, oocytes, microarray, in vitro maturation (IVM)

\section{INTRODUCTION}

The proper mammalian cumulus-oocyte complexes (COCs) maturation both in vivo and/or in vitro is a crucial step before successful monospermic fertilization. The process of oocytes maturation involves the cytoplasmic accumulation of mRNA, proteins and nuclear-chromosomal reorganization leading to the final stage, metaphase II. It was clearly demonstrated on several mammalian species, including pigs, that only fully mature gametes enable further growth and development of an embryo in periimplantation stages. Additionally, gap junction connections (GJC) facilitate proper communication between cells and cumulus-oocyte, which are important for female gamete maturation. It was shown that only gametes with proper bidirectional transport of metabolites are able to reach full growth. Our recent studies indicated that proteins called connexins, that form the GJC in porcine COCs, are expressed not only in cumulus cells, separated and/or enclosed, but also in isolated follicular granulosa cells (GCs). Hence, it is suggested that both oocyte's surrounding somatic cells and separated follicular cells are involved in several crucial metabolic/endocrinological associations and pathways.

The oocytes maturation is a compound process that involves numerous biochemical, metabolomic and morphologic changes, and is accompanied by expression of several clusters of genes. It was demonstrated in many mammalian species, and experimental models that expression of genes and proteins involved in transforming growth factor beta receptor (TGFBR) signaling pathway activation belongs to the main mechanisms responsible for regulation of organogenesis and morphogenesis. Furthermore, the proteins regulated by and/or associated with expression of TGFBR are crucial for cell survival and/or genetically programmed death. It was also shown that TGFBR-related peptides and enzymes are the main molecules involved in important biochemical and metabolomic processes associated with cell proliferation, differentiation, growth and development. So far, the role of TGFB and respective receptor in regulatory mechanisms of the oocyte, was demonstrated in mammals, including pigs. Moreover, the function of these genes and encoded proteins in the oocyte maturation capability, was also well recognized. However, this study is the first description regarding TGFBR-related cluster of genes transcriptomic profile in porcine immature and mature oocytes. 


\title{
MATERIAL AND METHODS
}

\begin{abstract}
ANIMALS
A total of 45 pubertal crossbred Landrace gilts, bred on a local, commercial farm, were used in this study. They had a mean age of 155 days (range $140-170$ days) and a mean weight of $100 \mathrm{~kg}(95-120 \mathrm{~kg})$. All of the animals were housed under identical conditions and fed the same forage (depending on age and reproductive status). The experiments were approved by the Local Ethic Committee.

\section{COLLECTION OF PORCINE OVARIES AND CUMULUS-OOCYTE-COMPLEXES (COCS)}

Ovaries and reproductive tracts were recovered at slaughter and transported to the laboratory at $38^{\circ} \mathrm{C}$ in $0.9 \% \mathrm{NaCl}$ within $40 \mathrm{~min}$. To provide optimal conditions for subsequent oocyte maturation and fertilization in vitro, the ovaries of each animal were placed in 5\% fetal bovine serum solution (FBS; Sigma-Aldrich Co., St. Louis, MO, USA) in PBS. Single large follicles $(>5 \mathrm{~mm})$ were then opened by puncturing with a $5 \mathrm{ml}$ syringe and $20-\mathrm{G}$ needle in a sterile Petri dish, and COCs were recovered. The COCs were washed three times in modified PBS supplemented with $36 \mu \mathrm{g} / \mathrm{ml}$ pyruvate, $50 \mu \mathrm{g} / \mathrm{ml}$ gentamycine, and $0.5 \mathrm{mg} / \mathrm{ml} \mathrm{BSA} \mathrm{(Sigma-Aldrich,}$ St. Louis, MO, USA). COCs were selected under an inverted microscope Zeiss, Axiovert 35 (Lübeck, Germany), counted, and morphologically evaluated using the scale suggested by Jackowska et al. [9]. Only COCs of grade I with homogeneous ooplasm and uniform, compact cumulus cells were considered for the following steps of the experiment, resulting 300 grade I oocytes $(3 \mathrm{x} n=50$ before IVM, $3 \mathrm{x}$ $\mathrm{n}=50$ after IVM).
\end{abstract}

\section{ASSESSMENT OF OOCYTE DEVELOPMENTAL COMPETENCE BY BCB TEST}

To perform the BCB staining test, oocytes were washed twice in modified Dulbecco PBS (DPBS) (Sigma-Aldrich, St. Louis, MO) supplemented with $50 \mathrm{IU} / \mathrm{ml}$ penicillin, $50 \mu \mathrm{g} / \mathrm{ml}$ streptomycin (Sigma-Aldrich, St. Louis, MO, USA), 0.4\% BSA $[\mathrm{w} / \mathrm{v}], 0.34 \mathrm{mM}$ pyruvate, and5.5 $\mathrm{mM}$ glucose (DPBSm). Thereafter, they were treated with $13 \mu \mathrm{M}$ BCB (Sigma-Aldrich, St. Louis, MO) diluted in DPBSm at $38.5^{\circ} \mathrm{C}$ 
and $5 \% \mathrm{CO}_{2}$ for $90 \mathrm{~min}$. After treatment, the oocytes were transferred to DPBSm and washed twice. During the washing procedure, the oocytes were examined under an inverted microscope and classified as either stained blue $\left(\mathrm{BCB}^{+}\right)$or remained colorless $\left(\mathrm{BCB}^{-}\right)$. Immature oocytes have compact cumulus cell layers that require removal for further oocyte evaluation. Next, the $\mathrm{BCB}^{+} \mathrm{COCs}$ were first incubated with bovine testicular hyaluronidase (Sigma-Aldrich, St. Louis, MO, USA) for 2 $\min$ at $38^{\circ} \mathrm{C}$ to separate cumulus and granulosa cells. Cells were then removed by vortexing the $\mathrm{BCB}^{+}$oocytes in $1 \%$ sodium citrate buffer followed by mechanical displacement using a small-diameter glass micropipette. Only the granulosa cell-free $\mathrm{BCB}^{+}$oocytes were used for subsequent IVM and microarray analysis.

\section{IN VITRO MATURATION OF PORCINE COCS}

After the first $\mathrm{BCB}$ test, the $\mathrm{COCs}$ which remained colorless (BCB-) were cultured in Nunclon ${ }^{\mathrm{TM}} \Delta 4$-well dishes in $500 \mu \mathrm{l}$ of standard porcine IVM culture medium TCM-199 (tissue culture medium) with Earle's salts and $L$-glutamine, (Gibco BRL Life Technologies, Grand Island, NY, USA) supplemented with $2.2 \mathrm{mg} / \mathrm{ml}$ sodium bicarbonate (Nacalai Tesque, Inc., Kyoto, Japan), $0.1 \mathrm{mg} / \mathrm{ml}$ sodium pyruvate (Sigma-Aldrich, St. Louis, MO, USA), $10 \mathrm{mg} / \mathrm{ml}$ BSA (bovine serum albumin), (Sigma-Aldrich, St. Louis, MO, USA), $0.1 \mathrm{mg} / \mathrm{ml}$ cysteine (Sigma-Aldrich, St. Louis, MO, USA), 10\% filtered porcine follicular fluid (v/v), and gonadotropin supplements at final concentrations of $2.5 \mathrm{IU} / \mathrm{ml} \mathrm{hCG}$ (Ayerst Laboratories, Inc., Philadelphia, PA, USA) and $2.5 \mathrm{IU} / \mathrm{ml} \mathrm{eCG}$ (Intervet, Whitby, ON, Canada). Wells were covered with a mineral oil overlay and cultured for 44 $\mathrm{h}$ at $38^{\circ} \mathrm{C}$ under $5 \% \mathrm{CO}_{2}$. After cultivation, the $\mathrm{BCB}$ staining test was performed again, and $\mathrm{BCB}^{+}$oocytes were used for further experiments.

\section{RNA EXTRACTION FROM PORCINE OOCYTES}

Oocytes investigated before and after in vitro maturation were pooled into three independent samples for each experimental group. Total RNA was extracted from samples using TRI Reagent (Sigma, St Louis, MO, USA) and RNeasy MinElute cleanup Kit (Qiagen, Hilden, Germany). The amount of total mRNA was determined from the optical density at $260 \mathrm{~nm}$, and the RNA purity was estimated using the 260/280 nm absorption ratio (higher than 1.8) (NanoDrop spectrophotometer, Thermo Scientific, ALAB, Poland). The RNA integrity and quality were checked on a Bioanalyzer 2100 (Agilent Technologies, Inc., Santa Clara, CA, USA). The resulting RNA integrity numbers (RINs) were between 8.5 and 10 with an avera- 
ge of 9.2 (Agilent Technologies, Inc., Santa Clara, CA, USA). The RNA in each sample was diluted to a concentration of $100 \mathrm{ng} / \mu \mathrm{l}$ with an OD260/OD280 ratio of 1.8/2.0. From each RNA sample, $500 \mathrm{ng}$ of RNA were taken.

\section{MICROARRAY EXPRESSION ANALYSIS AND STATISTICS}

The Affymetrix ${ }^{\circledR}$ procedure was previously described by Trejter et.al [22]. Total RNA (100 ng) from each pooled sample was subjected to two round, sense cDNA amplification (Ambion ${ }^{\circledR}$ WT Expression Kit). The obtained cDNA was used for biotin labeling and fragmentation by Affymetrix GeneChip ${ }^{\circledR}$ WT Terminal Labeling and Hybridization (Affymetrix ${ }^{\circledR}$ ). Biotin-labeled fragments of cDNA $(5.5 \mu \mathrm{g})$ were hybridized to the Affymetrix ${ }^{\circledR}$ Porcine Gene 1.1 ST Array Strip $\left(48^{\circ} \mathrm{C} / 20 \mathrm{~h}\right)$. Then, microarrays were washed and stained according to the technical protocol using the Affymetrix GeneAtlas ${ }^{\circledR}$ Fluidics Station. The array strips were scanned employing Imaging Station of the GeneAtlas ${ }^{\circledR}$ System. The preliminary analysis of the scanned chips was performed using Affymetrix GeneAtlas ${ }^{\circledR}$ Operating Software. The quality of gene expression data was confirmed according to the quality control criteria provided by the software. The obtained CEL files were imported into downstream data analysis software.

All of the presented analyses and graphs were performed using Bioconductor and $\mathrm{R}$ programming languages. Each CEL file was merged with a description file. In order to correct background, normalize, and summarize results, we used the Robust Multiarray Averaging (RMA) algorithm. To determine the statistical significance of the analyzed genes, moderated $t$-statistics from the empirical Bayes method were performed. The obtained p-value was corrected for multiple comparisons using Benjamini and Hochberg's false discovery rate. The selection of significantly altered genes was based on a p-value beneath 0.05 and expression higher than two fold.

Differentially expressed genes were subjected to the selection of genes involved in morphogenesis and cellular differentiation. The differentially expressed gene list (separated for up- and down-regulated genes) was uploaded to DAVID software (Database for Annotation, Visualization and Integrated Discovery), where genes belonging to "Transforming growth factor beta receptor signaling pathway" GO term were obtained. The term "Transforming growth factor beta receptor signaling pathway" (GO:0007179) is defined as "A series of molecular signals initiated by the binding of an extracellular ligand to a transforming growth factor beta receptor on the surface of a target cell, and ending with regulation of a downstream cellular process, e.g. transcription." It consists of 2307 anotated genes of which 126 are anotated to Susscrofa organism. 
Expression data of these genes were subjected to a hierarchical clusterization procedure, and their expression values were presented as a heat map. Besides predicting interactions DAVID software also allowed performance of functional enrichments of GO terms based on previously uploaded gene sets from the "Transforming growth factor beta receptor signaling pathway" GO BP term. The relation between genes that belongs to other GO terms were further investigated by GeneAnswers R package.

Interactions between differentially expressed genes/proteins belonging to "Transforming growth factor beta receptor signaling pathway" ontology group were investigated by STRING10 software (Search Tool for the Retrieval of Interacting Genes). The list of gene names was used as query for interaction prediction. The search criteria were based on co-occurrences of genes/proteins in scientific texts (text mining), co-expression, and experimentally observed interactions. The results of such analysis generated a gene/protein interaction network where the intensity of the edges reflected the strength of the interaction score. Two Genes with the strongest interactions from STRING analysis (Fos and SMAD4) were subjected to BioGraph web services. BioGraph is based on the integration of biomedical knowledge bases and yields intelligible and literature-supported indirect functional relations. A result of such analysis points to direct connections between two analyzed genes.

\section{RESULTS}

To investigate oocyte transcriptome changes after in vitro maturation in relation to transcriptome profile of freshly isolated oocyte, before in vitro procedure (in Vivo), We performed whole gene expression analysis by Affymetrix ${ }^{\circledR}$ Porcine Gene 1.1 ST Array. In such assay expression of more than 12258 porcine transcripts was examined. The genes for which the fold change was higher than the cut-off value $($ fold $>|2|)$ and corrected $\mathrm{p}$ value $(\operatorname{adj} \mathrm{p})<0.05$, were considered as differentially expressed. From the whole transcript that consist of 419 different genes, 379 genes were downregulated and 40 genes were up regulated in relation to the oocyte transcriptome before in vitro procedure.

Among these genes, genes belong to "Transforming growth factor beta receptor signaling pathway" gene ontology biological process term was extracted by DAVID (Database for Annotation, Visualization and Integrated Discovery) software. Up and down regulated gene sets were subjected to DAVID searching separately and only gene sets, where adj. $p$ value were lower than 0.05 were selected (tab. 1). This set of genes was subjected to hierarchical clusterization procedure and presented as heat map (fig. 1). 
TABLE 1. Fold changes, adjusted $p$ values and ENTREZ gene ID of differentially expressed genes belonging to the "Transforming growth factor beta receptor signaling pathway" functional category from DAVID GEOTERM BP database. Symbols and names of the selected genes are also shown

\begin{tabular}{c|c|c|c}
\hline NAME & FOLD & ADJ.P.VALUE & ENTREZ GENE ID \\
\hline MAP3K1 & 2.711751 & $2.474846 \mathrm{e}-02$ & 4214 \\
\hline ID1 & 2.980865 & $3.974331 \mathrm{e}-03$ & 3397 \\
\hline KLF10 & 2.466464 & $6.845130 \mathrm{e}-03$ & 7071 \\
\hline FOS & 18.941419 & $4.739475 \mathrm{e}-05$ & 2353 \\
\hline SMAD4 & 2.718853 & $1.238681 \mathrm{e}-03$ & 4089 \\
\hline TGFBR3 & 5.088482 & $4.059785 \mathrm{e}-04$ & 7049 \\
\hline
\end{tabular}

transforming growth factor beta receptor signaling pathway

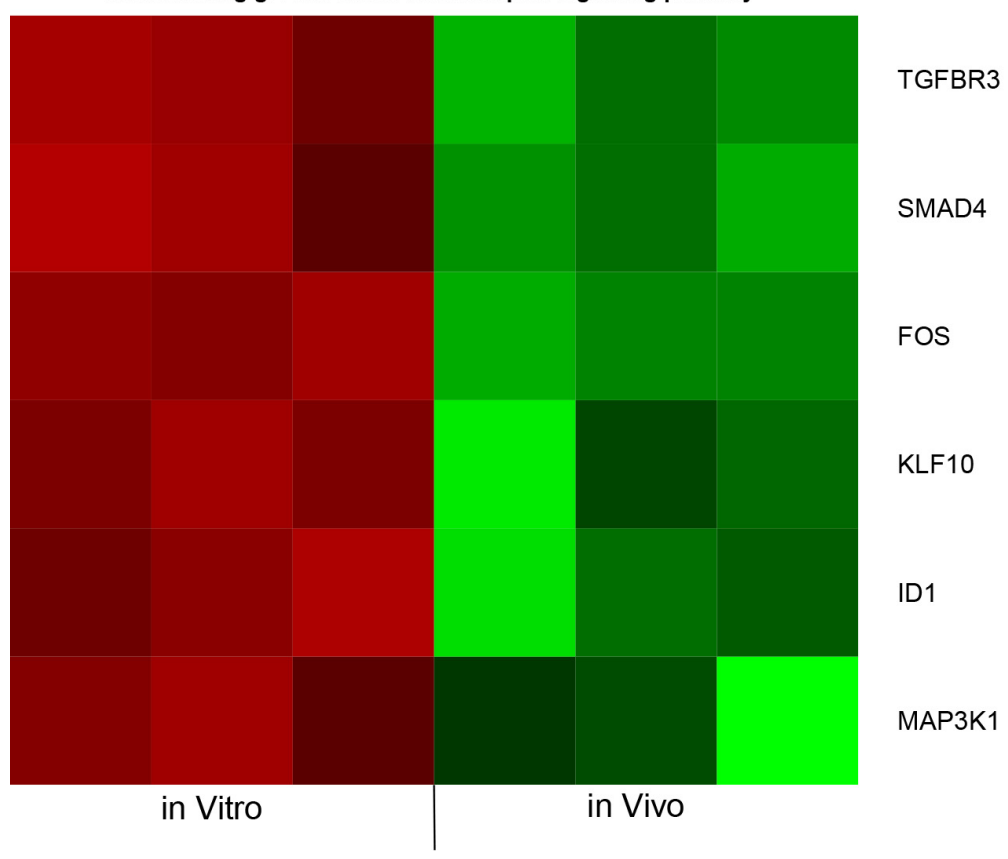

FIGURE 1. Heat map representation of differentially expressed genes belonging to the category "Transforming growth factor beta receptor signaling pathway" from GO.BP database. Arbitrary signal intensity acquired from microarray analysis is represented by colours (green, higher; red, lower expression). $\log 2$ signal intensity values were resized to Row Z-Score scale for any single genes (from -2 , the lowest expression to +2 , the highest expression) 
Genes from one particular GO group will belong also to other different GO term categories. By this reason we perform functional enrichments of GO terms based on previously uploaded gene set from "Transforming growth factor beta receptor signaling pathway" GO BP term. Top five GO terms that genes belong also to "Transforming growth factor beta receptor signaling pathway" were presented as table, where its category, term, number of genes that its share with "Positive regulation of macromolecule metabolic process", p-value and Benjamini - Hochberg's false discovery rate were shown (tab. 2). Categories were further investigated with GeneAnswers R package and the results were presented as concept-gene network (fig. 2).

TABLE 2. Top five GO categories formed by genes differentially expressed belonging to the "Transforming growth factor beta receptor signaling pathway" ontology group. GO categories were generated in STRING software. GO ID (pathway ID), GO term description (pathway description), number of the genes belonging to appropriate category (count in gene set) are shown

\begin{tabular}{c|c|c|c|c|c}
\hline \multicolumn{1}{c|}{ CATEGORY } & TERM & COUNT & $\%$ & P-VALUE & BENJAMINI \\
\hline GOTERM_BP_FAT & $\begin{array}{c}\text { transmembrane receptor protein serine/ } \\
\text { threonine kinase signaling pathway }\end{array}$ & 4 & 0,4 & $3,2 \mathrm{E}-5$ & $1,8 \mathrm{E}-2$ \\
\hline GOTERM_BP_FAT & $\begin{array}{c}\text { cellular response to growth factor } \\
\text { stimulus }\end{array}$ & 4 & 0,4 & $1,5 \mathrm{E}-4$ & $4,2 \mathrm{E}-2$ \\
\hline GOTERM_BP_FAT & response to growth factor & 4 & 0,4 & $1,6 \mathrm{E}-4$ & $3,0 \mathrm{E}-2$ \\
\hline GOTERM_BP_FAT & $\begin{array}{c}\text { enzyme linked receptor protein signa- } \\
\text { ling pathway }\end{array}$ & 4 & 0,4 & $3,9 \mathrm{E}-4$ & $5,5 \mathrm{E}-2$ \\
\hline GOTERM_BP_FAT & BMP signaling pathway & 3 & 0,3 & $5,4 \mathrm{E}-4$ & $5,1 \mathrm{E}-2$ \\
\hline
\end{tabular}

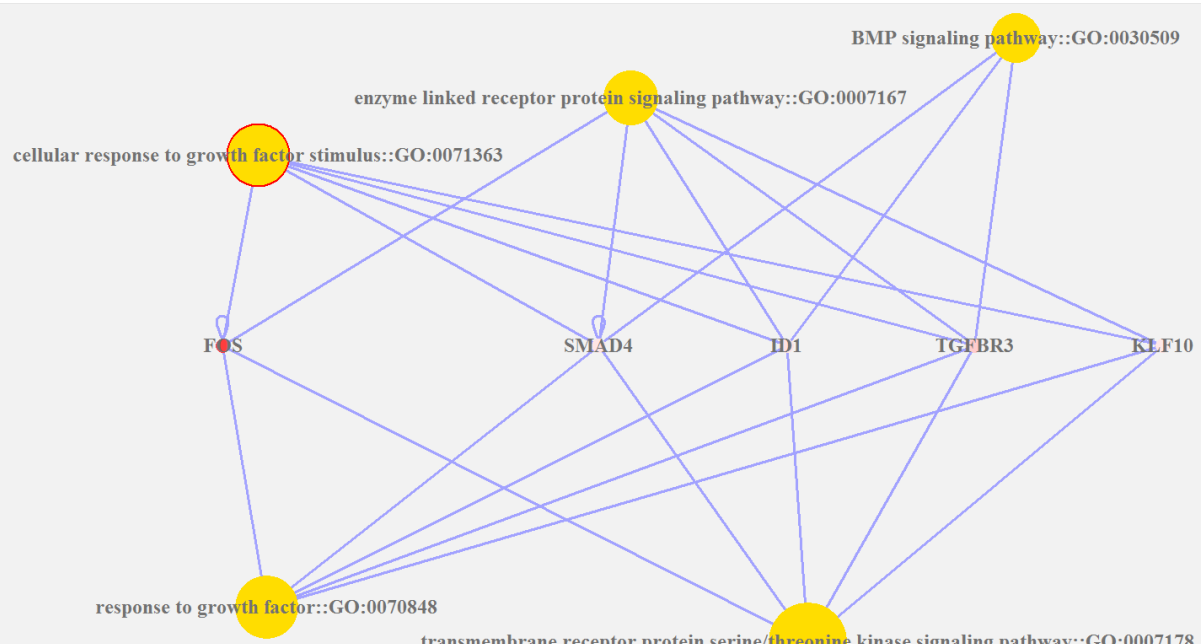

transmembrane receptor protein serine/threonine kinase signaling pathway::GO:0007178

FIGURE 2. Concept-gene network which shows which differently expressed genes from "Transforming growth factor beta receptor signaling pathway" belongs to which of top five GO category that shares genes with "Transforming growth factor beta receptor signaling pathway". The intensity of the red color of the dots represents the fold change 
STRING-generated interaction network was generated among differentially expressed genes belonging to the "Transforming growth factor beta receptor signaling pathway" term. Applied prediction methods used text mining, co-expression, and experimentally observed interactions (fig. 3). Based on that result relation between Fos and SMAD4 were further investigated by subjection to BioGraph web services. A result of this analysis has been shown on (fig. 4).
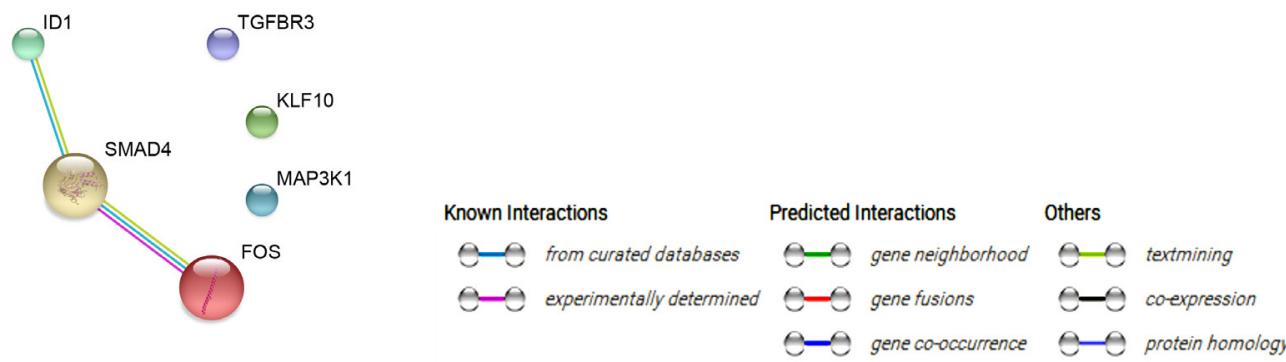

FIGURE 3. STRING-generated interaction network among differentially expressed genes belonging to the "Transforming growth factor beta receptor signaling pathway" ontology group. Applied prediction methods: text mining, co-expression, experimentally observed interactions

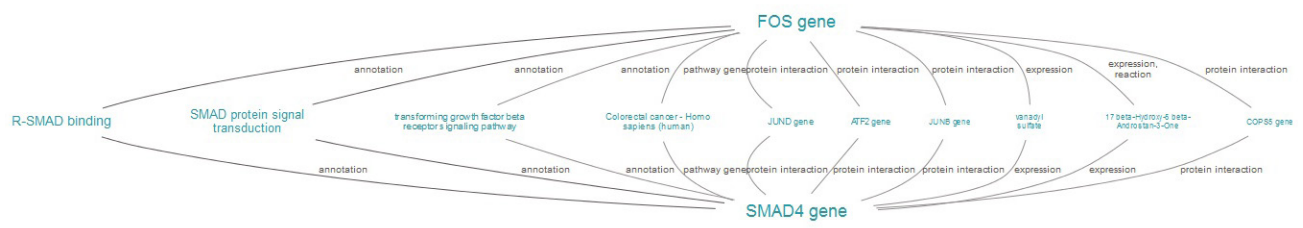

FIGURE 4. The representation of known interaction between Fos and SMAD4 proteins generated by biograph database

\section{DISCUSSION}

COCs maturation is characterized by considerable morphological and biochemical changes. Alterations can be observed both in vivo as well as in vitro maturation and among others involve changes in transcriptomic profile of genes up-regulated and down-regulated during COCs growth and development. TGFB and its receptors were found to play important role in process of oocytes maturation in pigs. However, the role of TGFB-related cluster genes and their transcriptomic profile in porcine immature and mature oocytes still needs further investigation.

In this study, we analyzed the transcriptomic profile of porcine oocytes before and after IVM. We found 6 genes involved in gamete maturation and identified transcripts with possible new function, which were: TGFBR3, SMAD4, FOS, KLF10, ID1 and MAP3K1. All of them decreased their expression after IVM. 
Majority of them, like TGFBR3, SMAD4, ID1 and MAP3K1 have been already described in relation to other ontological groups (our unpublished data).

Some of down-regulated genes correlated with the achievement of porcine oocytes MII stage were directly or indirectly associated with oocytes before maturation. One of them is SMAD4 (Smad Family Member 4) protein, which was shown to be activated in response to TGFB signaling. It forms homomeric and heteromeric complexes with other activated SMAD proteins (SMAD2/SMAD3-SMAD4), which subsequently regulate transcription of target genes by interaction with SMAD-binding element (SBE) in the nucleus. SMAD4 is a component of SMAD3/SMAD4/ JUN/FOS complex, forming AP1 promoter site, which is required for transcriptional activity in response to TGFB [8]. Mutations in SMAD4 gene were correlated with pancreatic cancer, therefore it is supposed it can act as a tumor suppressor. Furthermore, SMAD4 can be found in porcine ovary, mainly localized in the oocyte, granulosa and thecal cells at different stages of folliculogenesis, suggesting its relation to oocytes maturation or any other reproduction-related processes [25]. The generation of fully competent egg requires interactions between the oocyte and ovary, and TGFB was found to be major signaling pathway. Granulosa cells deprived of SMAD4 interrupt follicle growth, however its role in the oocyte has not been determined so far [11]. There are some information about TGFB signaling pathway involvement in sexual determination of germ cells. It was shown that SMAD4 signaling plays a key role in induction of oocyte-specific regulators and meiotic progression. It was proved by SMAD4 gene knock out, resulted in transformation of germ cells towards male pathway [24]. This phenomenon was characterized as somatic cells-independent. However, in the maturational stages of oocytes after birth, deletion of SMAD4 gene from granulosa cells was correlated with intensive, early luteinization and cumulus cells defects [16]. Additionally, SMAD4 and/or its downstream signaling regulates meiotic progression during oogenesis. It shows that SMAD4 function is stage-dependent in ovaries [24]. Similarly, in embryonic development SMAD4 was found to play crucial and stage-specific role, promoting preimplantation early embryonic development in mice [18]. Moreover, it is most likely species-specific function of oocyte-derived SMAD4 in promoting initial stages of embryogenesis, as it occurs earlier in bovine than in mice [13]. Conversely, Kaune et al., showed that SMAD4 and TGFB signaling in the oocyte, has no effect on oocyte or follicle development, ovulation, fertilization, preimplantation development, or implantation. They used mutant females with oocyte-specific SMAD4 deletion and control mice and mated them with control males. Examination showed no reduction in fertility nor litter size [11].

The FOS (Fos Proto-Oncogene, AP-1 Transcription Factor Subunit) gene is a component of previously mentioned SMAD3/SMAD4/JUN/FOS complex and its role in forming AP1 promoter site in response to TGFB stimulation was already described. The FOS was shown to play role in proliferation, differentiation 
and transformation, as well as in apoptosis, mainly of cells forming the skeleton. Furthermore, since AP1 is involved in early stages of follicle growth, it can be assumed that FOS has at least indirect input for this process [14]. It is presumed, that FOS mRNA is a potential target for mediating nuclear export MEX3C protein, which can regulate FOS by promoting its nuclear export. In mice, MEX3C transcripts are abundantly expressed in developing oocytes in the ovary, although are also expressed in granulosa cells and later stages of oocytes. Thus, all this suggest that MEX3C keeps stability of FOS mRNA in oocytes [12]. Therefore, down-regulated FOS expression after IVM can be promoted by decreased MEX3C expression and needs more advanced studies.

Subsequent factor, TGFBR3 (Transforming Growth Factor Beta Receptor 3) is one of TGFB membrane-bound co-receptors. TGFBR3 has the ability to capture TGFB, thus inhibiting TGFB signaling pathways. Down-regulated expression of TGFBR3 was observed in many types of cancers [3]. In the course of this study, we observed down-regulated expression of this gene in porcine COCs after IVM comparing to before IVM. It suggests that TGFBR3 is involved in maturation-related mechanism, however we cannot exclude its role in processes related to cumulus cells differentiation as well [1], since TGF/TGFB/TGFBR signaling cascade between oocytes and follicular cells maintains considerably higher activity at early stages of oogenesis and folliculogenesis. Thus, TGFB-TGFBR3 signaling in porcine oocytes and follicular cells may be essential for proper growth, development, and maturation.

The inhibitor of DNA binding (ID1), is another regulator of gene transcription. It binds to target transcription factors through its helix-loop-helix (HLH) motif. So far, ID1 was known to mediate cellular growth, development, proliferation, and differentiation [21], thus influencing cell survival, senescence, apoptosis, and angiogenesis $[6,23]$. Little is known about ID1 role in oocytes in vivo and in vitro maturation, however it is clear that this process also requires substantial proliferation and differentiation of cumulus cells. Blaha et al.studied the effect of FSH on IDI gene expression pattern in porcine cumulus cells with microarray assays. They found that FSH administration led to increased expression of IDI gene, and concluded that, since IDI is responsible for cumulus cells differentiation and processes leading to successful IVM, may be potentially marker gene of oocyte maturational competence in vitro [2]. Hogg et al. presented evidence that ID1 acts during embryogenesis and development promoting cell growth in sheep. Immunohistochemistry revealed expression of ID1 protein in granulosa and thecal cells of ovarian follicles during development, especially in granulosa cells adjacent to the oocyte. Alterations in ID1 expression pattern were associated with TGFB signaling, suggesting crucial ID1 role in the mammalian ovary [7]. Finally, Jeong et al., tested the influence of trichostatin A (TSA) on in vitro porcine embryo development. They found increased blastocysts yield after TSA treatment, however 
expression of IDI was down-regulated in in vitro group comparing to in vivo, irrespectively of TSA administration [10]. Thus, taking into account our results, we can assume ID1 expression can be favored in in vivo conditions.

However, there was also group of genes that in spite of wide spectrum of actions have never been associated with oocyte maturation or folliculogenesis. Previously they were correlated with other functions in mammalian organism, however they may be now recognized as new marker genes in processes of porcine folliculogenesis, oocytes maturation or embryonic developmental capacity. Among them KLF10 (Krüppel-like family) transcription factor attracts attention, since it was not implicated in reproduction processes so far. This TGFB-induced protein was associated with cell differentiation and regarded as potential marker for diseases in human, like breast cancer, cardiac hypertrophy, and osteoporosis [5, 17]. Furthermore, KLF10 can support TGFB inhibition of cell proliferation, inflammation, or even cause apoptosis. Moreover, it was shown on osteoblasts and pancreatic carcinoma cells examples that KLF10 overexpression substitutes the TGFB action. Binding to its target sequences KLF10 can both, activate of inhibit transcription of many genes, thus we cannot forejudge lack of its input in porcine reproductive processes [20].

The last analyzed gene was MAP3K1. Although the role of MAPK kinase in the process of folliculogenesis and oocyte maturation is well known, MAP3K1 contribution in these processes needs further investigation $[4,21]$. In this study, we describe the expression profile of MAP3K1 in porcine oocytes before or after IVM in relation to the TGFB signaling pathway, since there is no data indicating its role in mammalian oocyte maturation. MAPK-activated protein kinase 2 (MK2) was shown to influence meiotic competence in pigs, as lack of this protein resulted in spindle defects and arrested gametes at $\mathrm{GV}$ or the prometaphase $\mathrm{I} /$ metaphase I stage. MK 2 contributed also in organization of chromosome, microtubule, and kinetochore cellular architecture [15]. Interestingly, down-regulation of MAP3K1 after IVM suggested that it is not essential factor for oocyte maturation or other mentioned processes.

In conclusion, our data showed that TGFB signaling pathway-related genes were differentially expressed in oocytes and down-regulated after IVM compared to before IVM. Since their function in oocytes maturation was poorly investigated before, all analyzed genes need further investigation not only on transcript but also on protein level. It would be helpful in determining their potential as biomarkers for oocyte maturation and fertilization capability.

\section{ACKNOWLEDGEMENTS}

Publication of this article was made possible by grant number 2014/13/D/ NZ9/04798 "SONATA" from Polish National Centre of Science 


\section{REFERENCES}

[1] Al-Edani T, Assou S, Ferrières A, Bringer Deutsch S, Gala A, Lecellier CH, Aït-Ahmed O, HamAMAH S. Female aging alters expression of human cumulus cells genes that are essential for oocyte quality. Biomed Res Int. 2014; 2014: 964614.

[2] Blaha M, Nemcova L, Kepkova KV, Vodicka P, Prochazka R. Gene expression analysis of pig cumulus-oocyte complexes stimulated in vitro with follicle stimulating hormone or epidermal growth factor-like peptides. Reprod Biol Endocrinol. 2015; 13: 113.

[3] Brent A. Hanks, Alisha Holtzhausen, Katherine S. Evans, Reberah Jamieson, Petra Gimpel, Olivia M. Campbell, Melissa Hector-Greene, Lihong Sun, AlokTewari, Amanda George, Mark Starr, Andrew Nixon, Christi Augustine, Georgia Beasley, Douglas S. Tyler, TakayuOsada, Michael A. Morse, Leona Ling, H. Kim Lyerly, Gerard C. Blobe. Type III TGF- $\beta$ receptor downregulation generates an immunotolerant tumor microenvironment. J Clin Invest. 2013; 123(9): 3925-3940.

[4] Diaz FJ, O’Brien MJ, Wigglesworth K, Eppig JJ. The preantral granulosa cell to cumulus cell transition in the mouse ovary: development of competence to undergo expansion. Dev Biol. 2006; 299(1): 91-104.

[5] ElLENRIEDER V. TGFbeta regulated gene expression by Smads and Sp1/KLF-like transcription factors in cancer. Anticancer Res. 2008; 28: 1531-1539.

[6] Hara E, Yamaguchi T, Nojima H, Ide T, Campisi J, Okayama H, et al. Id-related genes encoding helix-loop-helix proteins are required for G1 progression and are repressed in senescent human fibroblasts. The Journal of biological chemistry. 1994; 269: 2139-45.

[7] Hogg K, Etherington SL, Young JM, McNeilly AS, Duncan WC. Inhibitor of differentiation (Id) genes are expressed in the steroidogenic cells of the ovine ovary and are differentially regulated by members of the transforming growth factor-beta family. Endocrinology. 2010; 151(3): 1247-56.

[8] Inman GJ, Nicolas FJ, Hill CS. Nucleocytoplasmic shuttling of Smads 2, 3, and 4 permits sensing of TGF-beta receptor activity. Molecular cell. 2002; 10: 283-94.

[9] Jackowska M, Kempisty B, Antosik P, Bukowska D, Budna J, Lianeri M, et al. The morphology of porcine oocytes is associated with zona pellucida glycoprotein transcript contents. Reproductive biology. 2009; 9: 79-85.

[10] Jeong YI, Park CH, Kim HS, Jeong YW, Lee JY, Park SW, Lee SY, Hyun SH, Kim YW, Shin T, Hwang WS. Effects of Trichostatin A on In vitro Development of Porcine Embryos Derived from Somatic Cell Nuclear Transfer. Asian-Australas J Anim Sci. 2013; 26(12): 1680-8

[11] Kaune H, Peyrache E, Williams SA. Oocyte-derived Smad4 is not required for development of the oocyte or the preimplantation embryo. Theriogenology. 2015; 83(5): 897-903.

[12] Li X, Li Y, Liu C, JIN M, Lu B. Oocyte-Specific Expression of Mouse MEX3C652AA in the Ovary and Its Potential Role in Regulating Maternal Fos mRNA. BiolReprod. 2016 May; 94(5):115.

[13] LI X, TRIPURANI SK, JAMES R, PANGAS SA. Minimal fertility defects in mice deficient in oocyte-expressed Smad4. BiolReprod. 2012; 86: 1-6.

[14] ОктАY KH, ОктАY MH. Immunohistochemical analysis of tyrosine phosphorylation and AP-1 transcription factors c-Jun, Jun D, and Fos family during early ovarian follicle development in the mouse. ApplImmunohistochemMolMorphol. 2004; 12(4): 364-9.

[15] Ou XH, Li S, Xu BZ, Chen LN, Jiang MX, Chen SQ, Chen NQ. Mitogen-activated protein kinase-activated protein kinase 2 is a critical regulator of pig oocyte meiotic maturation. ReprodFertil Dev. 2015:223-233

[16] Pangas SA, Li X, Robertson EJ, Matzuk MM. Premature luteinization and cumulus cell defects in ovarian-specific Smad4 knock out mice. MolEndocrinol. 2006; 20(6): 1406-22.

[17] Rajamannan NM, Subramaniam M, Abraham TP, Vasile VC, Ackerman MJ, Monroe DG, Chew TL, SPELSBERG TC. TGFbeta inducible early gene-1 (TIEG1) and cardiac hypertrophy: discovery and characterization of a novel signaling pathway. $J$ Cell Biochem. 2007; 100: 315-325. 
[18] Sirard C, de la Pompa Jl, Elia A, Itie A, Mirtsos C, Cheung A, Hahn S, Wakeham A, Schwartz L, Kern SE, Rossant J, MaK TW. The tumor suppressor gene Smad4/Dpc4 is required for gastrulation and later for anterior development of the mouse embryo. Genes Dev. 1998; 12: 107-119.

[19] Su YQ, Denegre JM, Wigglesworth K, Pendola FL, O’Brien MJ, Eppig JJ. Oocyte-dependent activation of mitogen-activated protein kinase (ERK1/2) in cumulus cells is required for the maturation of the mouse oocyte-cumulus cell complex. Dev Biol. 2003; 263(1): 126-38.

[20] Subramaniam M, Hawse JR, Rajamannan NM, Ingle JN, Spelsberg TC. Functional role of KLF10 in multiple disease processes. Biofactors. 2010; 36(1): 8-18.

[21] Sun XH. Constitutive expression of the Id1 gene impairs mouse B cell development. Cell. 1994; 79: 893-900.

[22] Trejter M, Hochol A, Tyczewska M, Ziolkowska A, Jopek K, Szyszka M, et al. Sex-related gene expression profiles in the adrenal cortex in the mature rat: microarray analysis with emphasis on genes involved in steroidogenesis. International journal of molecular medicine. 2015; 35: 702-14.

[23] Volpert OV, Pili R, Sikder Ha, Nelius T, Zaichuk T, Morris C, et al. Id1 regulates angiogenesis through transcriptional repression of thrombospondin-1. Cancer cell. 2002; 2: 473-83.

[24] Wu Q, Fukuda K, Kato Y, Zhou Z, Deng CX, Saga Y. Sexual Fate Change of XX Germ Cells Caused by the Deletion of SMAD4 and STRA8 Independent of Somatic Sex Reprogramming. PLoS Biol. 2016; 14(9): e1002553.

[25] Xing N, Liang Y, Gao Z, He J, He X, Li H, Dong C. Expression and localization of Smad2 and Smad4 proteins in the porcine ovary. ActaHistochem. 2014; 116(8): 1301-6.

Editor -

Bartosz Kempisty

Department of Histology and Embryology, Department of Anatomy

Poznań University of Medical Sciences

6 Święcickiego St., 60-781 Poznań, Poland

tel./fax: $+48618546418 /+48618546440$

e-mail: bkempisty@ump.edu.pl 